\title{
Development and validation of a predictive model for 90-day readmission following elective spine surgery
}

\author{
Scott L. Parker, MD, ${ }^{1}$ Ahilan Sivaganesan, MD, ${ }^{1}$ Silky Chotai, MD, ${ }^{1}$ Matthew J. McGirt, MD, ${ }^{2}$ \\ Anthony L. Asher, MD, ${ }^{2}$ and Clinton J. Devin, MD ${ }^{3}$ \\ Departments of ${ }^{1}$ Neurological Surgery and ${ }^{3}$ Orthopaedic Surgery, Vanderbilt University Medical Center, Nashville, Tennessee; \\ and ${ }^{2}$ Carolina Neurosurgery and Spine Associates, Charlotte, North Carolina
}

\begin{abstract}
OBJECTIVE Hospital readmissions lead to a significant increase in the total cost of care in patients undergoing elective spine surgery. Understanding factors associated with an increased risk of postoperative readmission could facilitate a reduction in such occurrences. The aims of this study were to develop and validate a predictive model for 90-day hospital readmission following elective spine surgery.

METHODS All patients undergoing elective spine surgery for degenerative disease were enrolled in a prospective longitudinal registry. All 90-day readmissions were prospectively recorded. For predictive modeling, all covariates were selected by choosing those variables that were significantly associated with readmission and by incorporating other relevant variables based on clinical intuition and the Akaike information criterion. Eighty percent of the sample was randomly selected for model development and $20 \%$ for model validation. Multiple logistic regression analysis was performed with Bayesian model averaging (BMA) to model the odds of 90-day readmission. Goodness of fit was assessed via the C-statistic, that is, the area under the receiver operating characteristic curve (AUC), using the training data set. Discrimination (predictive performance) was assessed using the C-statistic, as applied to the $20 \%$ validation data set.
\end{abstract}

RESULTS A total of 2803 consecutive patients were enrolled in the registry, and their data were analyzed for this study. Of this cohort, 227 (8.1\%) patients were readmitted to the hospital (for any cause) within 90 days postoperatively. Variables significantly associated with an increased risk of readmission were as follows (OR [95\% CI]): lumbar surgery 1.8 [1.1-2.8], government-issued insurance 2.0 [1.4-3.0], hypertension 2.1 [1.4-3.3], prior myocardial infarction 2.2 [1.2-3.8], diabetes 2.5 [1.7-3.7], and coagulation disorder 3.1 [1.6-5.8]. These variables, in addition to others determined a priori to be clinically relevant, comprised 32 inputs in the predictive model constructed using BMA. The AUC value for the training data set was 0.77 for model development and 0.76 for model validation.

CONCLUSIONS Identification of high-risk patients is feasible with the novel predictive model presented herein. Appropriate allocation of resources to reduce the postoperative incidence of readmission may reduce the readmission rate and the associated health care costs.

https://thejns.org/doi/abs/10.3171/2018.1.SPINE17505

KEYWORDS readmission; spine; degenerative; predictive; model

I $\mathrm{N}$ an attempt to curb the current unsustainable growth in health care costs, the Centers for Medicare and Medicaid Services (CMS) has initiated several cost containment and quality improvement measures. ${ }^{2}$ A very important example of these measures is the Hospital Readmissions Reduction Program (HRRP), which penalizes hospitals with relatively higher rates of Medicare readmissions. ${ }^{7,9}$ The Department of Health and Human Services (HHS) stated that the HRRP will play a significant role in its new goals, applying to most inpatient hospitals, to tie an increasing share of traditional Medicare payments to quality or value in the coming years. Private payers are also developing an interest in this important metric. ${ }^{10,15}$ Many health care quality scientists believe readmissions are a strong indicator of a fragmented health care system that fails to identify patients at high risk for readmission and that too often leaves discharged patients confused about how to care for themselves at home and obtain necessary follow-up care. Additionally, readmissions are costly; Medicare alone reports spending $\$ 17.8$ billion a year on

ABBREVIATIONS ASA = American Association of Anesthesiologists; AUC = area under the receiver operating characteristic curve; BMA = Bayesian model averaging; CMS = Centers for Medicare and Medicaid Services; HRRP = Hospital Readmissions Reduction Program.

SUBMITTED May 1, 2017. ACCEPTED January 24, 2018.

INCLUDE WHEN CITING Published online June 15, 2018; DOI: 10.3171/2018.1.SPINE17505. 
patients whose return trips to the hospital could have been avoided. ${ }^{3,6}$ As part of the HRRP, the CMS is currently collecting data that may be used to financially penalize institutions based on high readmission rates after total joint arthroplasty. ${ }^{2}$ This policy may soon extend to other surgical procedures, including spine surgery.

Hospital readmissions following spine surgery can dramatically raise costs and reduce the overall value of surgical spine care; thus, a better understanding of the factors associated with readmission after spine surgery is needed to facilitate efforts aimed at quality improvement. Prior research into postoperative spine readmissions has relied heavily on administrative databases and thus has been restricted by the inherent limitations of such analyses. Nevertheless, these prior investigations have found rates of 30-day readmission after spine surgery ranging from $2.5 \%$ to $8.4 \%{ }^{4,8,11,12,14-16}$ These studies have identified the most common causes of readmission including woundrelated complications, infections, pain, and thromboembolic events; however, the identification and importance of specific risk factors for hospital readmission have yet to be elucidated. To date, there has been no comprehensive prospective analysis with the primary intent of understanding factors associated with hospital readmission following spine surgery. Therefore, we set out 1) to understand the factors associated with unplanned hospital readmission following elective spine surgery and 2) to develop a predictive model that could estimate the risk of 90-day readmission based on data obtained from our institutional prospective, longitudinal spine registry.

\section{Methods}

All patients undergoing elective spinal surgery for degenerative disease in the period between 2010 and 2014 at a single academic, comprehensive spine center were entered into a prospective, web-based longitudinal registry. Inclusion criteria for the present study were as follows: 1) patients with an age $>18$ years, 2) presenting symptoms with correlative imaging findings for diagnosis, 3) 3 months of failed multimodal conservative care, and 4) progressive neurological deficits.

Prospectively collected preoperative variables included patient demographics, comorbidities, diagnoses, and radiographic findings. Length of surgery, estimated blood loss, perioperative complications, and length of hospital stay were also prospectively recorded. Hospital readmission during the 90-day global period was recorded, as was a return to the operating room during this time. All morbidity and death occurring within the first 3 months after surgery were prospectively recorded.

Reasons for readmission were stratified into four categories: 1) medical, 2) wound related, 3) surgical failure, and 4) uncontrollable pain. Medically necessitated readmissions included those attributed to electrolyte abnormalities, persistent nausea/vomiting, cardiac arrhythmias, urinary retention, renal insufficiency, non-wound infections (urinary tract infection, pneumonia), uncontrolled diabetes, pulmonary embolus, deep vein thrombosis, and ileus. Wound-related readmissions included infection, dehiscence, pseudomeningocele, cerebrospinal fluid leak, and hematoma. Surgical failure readmissions included cases of persistent symptoms from inadequate decompression, hardware failure, and new neurological deficits.

\section{Statistical Analysis}

Descriptive statistics were conducted, and the results were expressed as the mean \pm standard deviation, compared via the Student t-test, and as the median (interquartile range), compared via the Mann-Whitney U-test. Nominal data were compared via the chi-square test. A p value $<0.05$ was considered statistically significant. Multivariable logistic regression analysis was performed to determine factors associated with 90 -day hospital readmission.

The first step in developing the predictive model for readmission was variable selection. This was done by choosing those variables that were found to be significantly associated with readmission and by incorporating other relevant variables based on clinical intuition and the Akaike information criterion (AIC). The available data were randomly partitioned into an $80 \%$ "training" data set and a 20\% "validation" data set, while ensuring that the incidence of readmission remained equal between the two groups. The selected predictor variables were then included in a multivariable logistic regression model, which was fit using the training data set. Because our goal was to optimize prediction while properly accommodating model uncertainty, the logistic regression was fitted using Bayesian model averaging (BMA). To assess predictive performance, the model was then used to predict the odds of a 90-day readmission for patients in the validation data set. Area under the receiver operating characteristic curve (AUC) analysis was conducted to quantify goodness of fit and the predictive performance of the model. All statistical analyses were conducted using SPSS version 20 (IBM Corp.) and R.

\section{Results}

\section{Patient Characteristics}

A total of 2803 consecutive patients were enrolled in our institutional registry over the study time period. Of this cohort, $227(8.1 \%)$ patients were readmitted to the hospital (for any cause) within 90 days postoperatively. The primary reason for readmission was medical for 120 (52.9\%) patients, wound related for $72(31.7 \%)$ patients, surgical failure for $27(11.9 \%)$ patients, and uncontrollable pain for $8(3.5 \%)$ patients.

Patients requiring readmission were older $(59.5 \pm 12.8$ years vs $56.3 \pm 13.7$ years, $\mathrm{p}=0.0004)$ and more likely to have governmental (Medicare/Medicaid) insurance (55.5\% vs $45.2 \%, p=0.004)$, a higher-risk anesthesia classification (American Society of Anesthesiologists [ASA] class III-IV: $78.4 \%$ vs $65.0 \%$, $\mathrm{p}<0.0001)$, and various medical comorbidities ( $\mathrm{p}$ ranges from $<0.0001$ to 0.04 ) than the patients without a hospital readmission (Table 1). A diagnosis of pseudarthrosis $(10.1 \%$ vs $6.1 \%, \mathrm{p}=0.02)$ and tumor $(8.4 \%$ vs. $4.1 \%, \mathrm{p}=0.006)$ was more likely to be present in the patient cohort requiring readmission, whereas a diagnosis of herniated disc $(18.9 \%$ vs $26.4 \%$, p $=0.01$ ) was less often present in the readmission versus the non-readmission cohort. 
TABLE 1. Baseline variables for patients with and without 90 -day readmission following elective spine surgery

\begin{tabular}{|c|c|c|c|}
\hline Parameter & $\begin{array}{c}\text { 90-Day } \\
\text { Readmission }\end{array}$ & $\begin{array}{l}\text { No } \\
\text { Readmission }\end{array}$ & $\begin{array}{c}p \\
\text { Value }\end{array}$ \\
\hline No. of patients & 227 & 2576 & \\
\hline Age in yrs & $59.5 \pm 12.8$ & $56.3 \pm 13.7$ & 0.0004 \\
\hline Male & $119(52.4 \%)$ & $1282(49.8 \%)$ & 0.45 \\
\hline \multicolumn{4}{|l|}{ Race } \\
\hline White & $208(91.6 \%)$ & $2280(88.5 \%)$ & 0.19 \\
\hline African American & $16(7.0 \%)$ & 206 (8.0\%) & 0.70 \\
\hline Other & $3(1.3 \%)$ & $90(3.5 \%)$ & 0.08 \\
\hline \multicolumn{4}{|l|}{ Insurance status* } \\
\hline Private & $95(41.9 \%)$ & $1345(52.2 \%)$ & 0.003 \\
\hline Governmental & $126(55.5 \%)$ & $1165(45.2 \%)$ & 0.004 \\
\hline None & $5(2.2 \%)$ & $61(2.4 \%)$ & 1.0 \\
\hline \multicolumn{4}{|l|}{ Diagnosis* } \\
\hline Stenosis & $72(31.7 \%)$ & $833(32.3 \%)$ & 0.88 \\
\hline Spondylolisthesis & $35(15.4 \%)$ & $533(20.7 \%)$ & 0.06 \\
\hline Herniated disc & $43(18.9 \%)$ & $681(26.4 \%)$ & 0.01 \\
\hline Pseudarthrosis & $23(10.1 \%)$ & $157(6.1 \%)$ & 0.02 \\
\hline Deformity/scoliosis & $22(9.7 \%)$ & $162(6.3 \%)$ & 0.07 \\
\hline Fracture & $13(5.7 \%)$ & $90(3.5 \%)$ & 0.10 \\
\hline Tumor & $19(8.4 \%)$ & $106(4.1 \%)$ & 0.006 \\
\hline Infection & $0(0.0 \%)$ & $12(0.5 \%)$ & 0.62 \\
\hline \multicolumn{4}{|l|}{ Symptom duration } \\
\hline$<3$ mos & $35(15.4 \%)$ & $335(13.0 \%)$ & 0.31 \\
\hline $3-12$ mos & $79(34.8 \%)$ & $921(35.8 \%)$ & 0.83 \\
\hline$>12$ mos & $113(49.8 \%)$ & $1320(51.2 \%)$ & 0.68 \\
\hline Preop narcotic use & $140(61.7 \%)$ & $1445(56.1 \%)$ & 0.11 \\
\hline \multicolumn{4}{|l|}{ ASA class* } \\
\hline I & $2(0.9 \%)$ & $63(2.5 \%)$ & 0.17 \\
\hline$\|$ & $46(20.3 \%)$ & $816(31.7 \%)$ & 0.0003 \\
\hline III & $163(71.8 \%)$ & $1619(62.9 \%)$ & 0.008 \\
\hline IV & $15(6.6 \%)$ & $53(2.1 \%)$ & 0.0002 \\
\hline CAD & $57(25.1 \%)$ & $474(18.4 \%)$ & 0.02 \\
\hline HTN & $158(69.6 \%)$ & $1410(54.7 \%)$ & $<0.0001$ \\
\hline $\mathrm{Ml}$ & $22(9.7 \%)$ & $108(4.2 \%)$ & 0.0007 \\
\hline Atrial fibrillation & $20(8.8 \%)$ & $101(3.9 \%)$ & 0.002 \\
\hline $\mathrm{CHF}$ & $13(5.7 \%)$ & $64(2.5 \%)$ & 0.009 \\
\hline COPD & $14(6.2 \%)$ & $86(3.3 \%)$ & 0.04 \\
\hline Diabetes & $71(31.3 \%)$ & $528(20.5 \%)$ & 0.003 \\
\hline Osteoporosis & $12(5.3 \%)$ & $74(2.9 \%)$ & 0.07 \\
\hline $\mathrm{BMI}$ in $\mathrm{kg} / \mathrm{m}^{2}$ & $30.2 \pm 7.3$ & $30.2 \pm 7.1$ & 0.97 \\
\hline Current smoker & $58(25.6 \%)$ & $550(21.4 \%)$ & 0.15 \\
\hline Preop anticoagulation & $19(8.4 \%)$ & $53(2.1 \%)$ & $<0.0001$ \\
\hline
\end{tabular}

$\mathrm{CAD}=$ coronary artery disease; $\mathrm{CHF}=$ congestive heart failure; $\mathrm{COPD}=$ chronic obstructive pulmonary disease; $\mathrm{HTN}=$ hypertension; $\mathrm{MI}=$ myocardial infarction.

Values expressed as the mean \pm standard deviation or as frequency (\%), unless indicated otherwise.

* Data not available in all cases.

\section{Operative and Perioperative Variables}

Patients in the readmission cohort were more likely to have surgery in the lumbar spine $(77.5 \%$ vs $69.8 \%, \mathrm{p}=$ $0.02)$, revision surgery ( $34.8 \%$ vs $27.4 \%, \mathrm{p}=0.02)$, fusion
TABLE 2. Operative and perioperative variables in patients with and without 90 -day readmission after elective spine surgery

\begin{tabular}{lccc}
\hline \multicolumn{1}{c}{ Parameter } & $\begin{array}{c}90 \text {-Day } \\
\text { Readmission }\end{array}$ & $\begin{array}{c}\text { No } \\
\text { Readmission }\end{array}$ & $\begin{array}{c}\mathrm{p} \\
\text { Value }\end{array}$ \\
\hline No. of patients & 227 & 2576 & \\
\hline $\begin{array}{l}\text { Site of surgery } \\
\text { Cervical/thoracic }\end{array}$ & $51(22.5 \%)$ & $779(30.2 \%)$ & 0.02 \\
$\quad$ Lumbar & $176(77.5 \%)$ & $1797(69.8 \%)$ & \\
\hline Revision surgery & $79(34.8 \%)$ & $705(27.4 \%)$ & 0.02 \\
\hline Fusion surgery & $152(67.0 \%)$ & $1159(45.0 \%)$ & $<0.0001$ \\
\hline Posterior approach & $200(88.1 \%)$ & $1884(73.1 \%)$ & $<0.0001$ \\
\hline No. of operative levels & $2[1-3.5]$ & $2[1-3]$ & 0.65 \\
\hline Length of surgery in mins & $199[136.5-274]$ & $173[118-246]$ & 0.001 \\
\hline Length of stay in days & $3[2-5]$ & $1[2-4]$ & 0.24 \\
\hline
\end{tabular}

Values expressed as frequency (\%) or as median [interquartile range], unless indicated otherwise.

procedures $(67.0 \%$ vs $45.0 \%, \mathrm{p}<0.0001)$, and posterior approaches $(88.1 \%$ vs $73.1 \%, \mathrm{p}<0.0001)$ than were patients in the non-readmission cohort (Table 2). The median number of operative levels was similar between the readmission and non-readmission cohorts (2 [1-3.5] levels vs 2 [1-3] levels, $p=0.65$ ); however, patients in the readmission cohort did have longer surgeries than the non-readmission cohort (199 [136.5-274] minutes vs 173 [118-246] minutes, $\mathrm{p}=0.001$ ). There was no difference in the length of hospitalization between the readmission and non-readmission cohorts $(3[2-5]$ days vs $1[2-4]$ days, $\mathrm{p}=0.24)$.

\section{Multivariable Analysis and the Predictive Model}

Variables that remained significantly associated with hospital readmission in the multivariable logistic regression analysis were as follows (OR [95\% confidence interval]): lumbar surgery 1.8 [1.1-2.8], government-issued insurance 2.0 [1.4-3.0], hypertension 2.1 [1.4-3.3], prior myocardial infarction 2.2 [1.2-3.8], diabetes 2.5 [1.7-3.7], and coagulation disorder 3.1 [1.6-5.8] (Table 3).

The full list of 32 variables included in the predictive model appears in Table 4. Variables used to create the model included those that were found to be significant in the multivariable logistic regression as well as other relevant variables as determined by clinical intuition and the Akaike information criterion. The AUC for the model, as applied to the training data set, was found to be 0.77 (representing goodness of fit). When the model was applied to the validation data set, an AUC of 0.76 (predictive performance) was achieved (Fig. 1).

\section{Discussion}

In a retrospective longitudinal cohort study, we collected data on demographics, presentation, surgical variables, and the need for readmission within 90 days postoperatively for patients undergoing elective spinal surgery at our institution. In this study of 2803 consecutive patients, we observed a 90-day readmission rate of $8.1 \%$. The most common reason for readmission during this period was medical (52.9\%). The remaining readmissions were secondary to 
TABLE 3. Variables that remained significantly associated with hospital readmission in a multiple logistic regression analysis

\begin{tabular}{ll}
\hline \multicolumn{1}{c}{ Variable } & OR $(95 \% \mathrm{Cl})$ \\
\hline Lumbar surgery & $1.8(1.1-2.8)$ \\
\hline Government-issued insurance & $2.0(1.4-3.0)$ \\
\hline Hypertension & $2.1(1.4-3.3)$ \\
\hline History of MI & $2.2(1.2-3.8)$ \\
\hline Diabetes & $2.5(1.7-3.7)$ \\
\hline History of coagulation disorder & $3.1(1.6-5.8)$ \\
\hline
\end{tabular}

wound complications (31.7\%), surgical failure (11.9\%), or uncontrollable pain (3.5\%). Variables found to be significantly associated with 90-day readmission following spine surgery in a multiple logistic regression analysis included lumbar surgery, government-issued insurance, hypertension, prior myocardial infarction, diabetes, and coagulation disorder. Using the factors found to be associated with readmission, we created a novel model to predict an individual patient's odds of readmission to the hospital within 90 days of spine surgery. When tested on a validation cohort, this model was able to accurately predict the occurrence of readmission with good validity ( $\mathrm{AUC}=0.76$ ).

Prior research into postoperative spine readmissions has largely been confined to administrative databases. All of these studies are retrospective in nature and are subject to limitations inherent to the use of administrative databases. Wang et al. queried the Medicare claims database and found 30-day readmission rates of $7.9 \%$ for cervical surgery and $7.3 \%$ for lumbar surgery. ${ }^{16}$ Factors found to be associated with a higher risk of readmission in their analysis were older age, greater number of comorbidities, dual eligibility for Medicare/Medicaid, and a greater number of fused levels. In our analysis, governmentissued insurance was associated with a higher likelihood of readmission compared to private insurance. The older age and associated comorbidities in the Medicare group and limited resources in indigent patients can explain the higher readmission rates in these groups. Kim et al. reviewed the National Surgical Quality Improvement Program (NSQIP) database for patients undergoing lumbar decompression and found a $4.4 \%$ rate of readmission. ${ }^{8}$ Factors significantly associated with readmission in that analysis were anemia, dependent functional status, operative duration, higher ASA classification, and occurrence of postoperative complications. In our analysis, comorbidities including hypertension, prior myocardial infarction, diabetes, and coagulation disorder were associated with higher chances of readmission. Patients with these comorbidities may need additional outpatient care including close follow-up, diagnosis, and management during the perioperative period to minimize the likelihood of readmission. Furthermore, these patients can be considered a high-risk group and should be incorporated differently into the episode-of-care health care model so that their additional costs of readmission can be covered.

The novel statistical model presented in this study was able to predict a patient's risk of postoperative readmission with good validity $(\mathrm{AUC}=0.76)$. We utilized a pro-
TABLE 4. Full list of 32 variables included in the predictive model

\begin{tabular}{ll}
\hline & Predictor Variables \\
\hline White race & History of coagulation disorder \\
\hline Current smoker & Governmental insurance \\
\hline History of CAD & Diagnosis \\
\hline History of HTN & Preoperative narcotic use \\
\hline History of MI & Symptom duration \\
\hline History of atrial fibrillation & Lumbar surgery \\
\hline History of diabetes & Revision surgery \\
\hline History of osteoporosis & Length of surgery \\
\hline Age & Ambulation \\
\hline Deformity/scoliosis & Pseudarthrosis \\
\hline Spinal tumor & Motor deficit \\
\hline Number of operative levels & Estimated blood loss \\
\hline Length of surgery & ASA classification \\
\hline History of CHF & History of COPD \\
\hline History of arthritis & BMI \\
\hline Baseline anxiety & Baseline depression \\
\hline
\end{tabular}

spective spine registry to observe the incidence of 90-day readmission following elective spinal surgery at our institution. Clinical registries use observational study methods to collect uniform data to evaluate specified outcomes for a defined population. ${ }^{5}$ The primary advantage of welldesigned registries from a scientific perspective relates to their strong external validity, which is achieved through an inclusive design that seeks to evaluate heterogeneous populations. As a result of this, the observed outcomes from registry analyses are often more representative of what is achieved in real-world practice and can more reliably be generalized to broad patient populations. ${ }^{1}$ Regular use of such a model can lead to more informed decision-making when discussing treatment options and expectations with

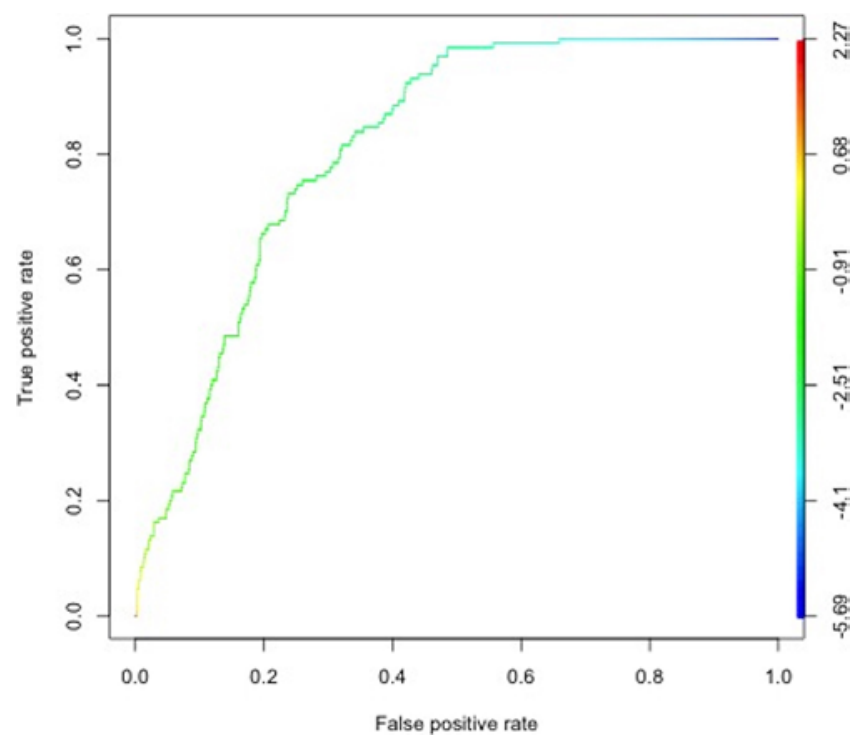

FIG. 1. The AUC for the 90-day readmission model. Figure is available in color online only. 
patients. High-risk patients requiring surgery can be prospectively identified, and appropriate interventions can be instituted both pre- and immediately postoperatively to help reduce the incidence of unplanned readmission. Analyses such as these can also allow hospitals and surgeons to risk stratify their practices to allow for more appropriate third party evaluations of patient outcomes. Furthermore, understanding and accurately predicting which patients may require additional resource utilization within a global period after surgery may help to facilitate the creation and implementation of risk-adjusted bundled payment systems that would more fairly compensate surgeons and hospitals for advanced services.

There are limitations to our study that have implications for its interpretation. In particular, the results represent a single institutional perspective. Therefore, medical centers with varying patient demographics, surgical case indexes, and practice patterns may have different rates of readmission and causative associations. To date there have been no prospective multicenter analyses of postoperative spine surgery readmission rates inclusive of all adult ages and insurance types-factors critical to generalizability. Data obtained from emerging national registry efforts in organized neurosurgery such as the Quality Outcomes Database (QOD) will hopefully help to bridge this knowledge gap.

\section{Conclusions}

Several pre- and perioperative factors were found to be associated with readmission following elective spine surgery. This study demonstrates that identification of highrisk patients is feasible. Furthermore, a novel predictive model derived from these data was able to accurately predict 90-day readmission with a sensitivity and specificity of $76 \%$. Identification of high-risk patients and appropriate allocation of resources to reduce the postoperative incidence of readmission may reduce its rate and associated health care costs.

\section{References}

1. Asher AL, Parker SL, Rolston JD, Selden NR, McGirt MJ: Using clinical registries to improve the quality of neurosurgical care. Neurosurg Clin N Am 26:253-263, ix-x, 2015

2. Centers for Medicare and Medicaid Services: Hospitalwide all-cause unplanned readmission (HWR) measure. QualityNet.org. (https://www.qualitynet.org/dcs/ ContentServer?c=Page\&pagename $=$ QnetPublic $\% 2 \mathrm{FPage} \% 2$ FQnetTier4\&cid=1228772504318) [Accessed March 1, 2018]

3. Centers for Medicare and Medicaid Services: National Health Expenditure Projections 2011-2021. Baltimore: Centers for Medicare and Medicaid Services, 2009 (https:// www.cms.gov/Research-Statistics-Data-and-Systems/ Statistics-Trends-and-Reports/NationalHealthExpendData/) [Accessed March 1, 2018]

4. Dailey EA, Cizik A, Kasten J, Chapman JR, Lee MJ: Risk factors for readmission of orthopaedic surgical patients. J Bone Joint Surg Am 95:1012-1019, 2013

5. Gliklich RE, Dreyer NA, Leavy MB (eds): Registries for Evaluating Patient Outcomes, 3rd edition. A User's Guide. Rockville, MD: Agency for Healthcare Research and Quality, 2014 (https://www.ncbi.nlm.nih.gov/books/ NBK208616/) [Accessed February 16, 2018]
6. Hines AL, Barrett ML, Jiang HJ, Steiner CA: Conditions with the Largest Number of Adult Hospital Readmissions by Payer, 2011. HCUP Statistical Brief \#172. Rockville, MD: Agency for Healthcare Research and Quality, 2014. (https://www.hcup-us.ahrq.gov/reports/statbriefs/ sb172-Conditions-Readmissions-Payer.jsp) [Accessed February 16, 2018]

7. Jencks SF, Williams MV, Coleman EA: Rehospitalizations among patients in the Medicare fee-for-service program. $\mathbf{N}$ Engl J Med 360:1418-1428, 2009

8. Kim BD, Smith TR, Lim S, Cybulski GR, Kim JY: Predictors of unplanned readmission in patients undergoing lumbar decompression: multi-institutional analysis of 7016 patients. J Neurosurg Spine 20:606-616, 2014

9. Kocher RP, Adashi EY: Hospital readmissions and the Affordable Care Act: paying for coordinated quality care. JAMA 306:1794-1795, 2011

10. Lohr KN, Schroeder SA: A strategy for quality assurance in Medicare. N Engl J Med 322:707-712, 1990

11. Lovecchio F, Hsu WK, Smith TR, Cybulski G, Kim B, Kim JY: Predictors of thirty-day readmission after anterior cervical fusion. Spine (Phila Pa 1976) 39:127-133, 2014

12. McCormack RA, Hunter T, Ramos N, Michels R, Hutzler L, Bosco JA: An analysis of causes of readmission after spine surgery. Spine (Phila Pa 1976) 37:1260-1266, 2012

13. Medicare Payment Advisory Commission: Report to the Congress: Reforming the Delivery System. Washington, DC: Medicare Payment Advisory Commission, 2008 (http:// www.medpac.gov/docs/default-source/reports/Jun08_ EntireReport.pdf) [Accessed March 1, 2018]

14. Pugely AJ, Callaghan JJ, Martin CT, Cram P, Gao Y: Incidence of and risk factors for 30-day readmission following elective primary total joint arthroplasty: analysis from the ACS-NSQIP. J Arthroplasty 28:1499-1504, 2013

15. Schairer WW, Carrer A, Deviren V, Hu SS, Takemoto S, Mummaneni P, et al: Hospital readmission after spine fusion for adult spinal deformity. Spine (Phila Pa 1976) 38:16811689,2013

16. Wang MC, Shivakoti M, Sparapani RA, Guo C, Laud PW, Nattinger AB: Thirty-day readmissions after elective spine surgery for degenerative conditions among US Medicare beneficiaries. Spine J 12:902-911, 2012

\section{Disclosures}

Dr. Devin has been a consultant for and has received support from Stryker Spine for non-study-related clinical or research effort. Dr. McGirt has been a consultant for Stryker.

\section{Author Contributions}

Conception and design: Devin, Parker, Sivaganesan, McGirt, Asher. Acquisition of data: Chotai. Analysis and interpretation of data: Parker, Sivaganesan, Chotai. Drafting the article: Parker, Sivaganesan. Critically revising the article: Devin, Parker, Sivaganesan, McGirt, Asher. Reviewed submitted version of manuscript: all authors. Statistical analysis: Sivaganesan. Administrative/technical/material support: Chotai. Study supervision: Devin, Parker, McGirt, Asher.

\section{Supplemental Information}

\section{Previous Presentations}

The abstract was presented as a podium presentation at the 30th Annual Meeting of the North American Spine Society held in Chicago, IL, on October 14-17, 2015.

\section{Correspondence}

Clinton J. Devin: Vanderbilt University Medical Center, Nashville, TN. clinton.j.devin@vanderbilt.edu. 